\title{
Resonance Dynamics and Decoherence
}

\author{
Marco Merkli
}

\begin{abstract}
We present a rigorous analysis of the phenomenon of decoherence for general $N$-level quantum systems coupled a reservoir modelled by a thermal bosonic quantum environment. We present an explicit form of the dominant reduced dynamics of open systems. We give explicit results for a spin $1 / 2$ (qubit), including decoherence and thermalization times. Our approach is based on a dynamical theory of quantum resonances. It yields the exact reduced dynamics of the small system and does not involve master equation or van Hove limit approximations. This approach is suitable for a wide variety of systems which are not explicitly solvable, including systems of interacting spins (registers of interacting qubits), for which the coupling between the system and the environment is fixed but small.
\end{abstract}

Mathematics Subject Classification (2000). Primary 82C10; Secondary 37L05.

Keywords. Decoherence, open quantum systems, reduced density matrix, thermal environment, spins, qubits, classical-quantum transition, non-equilibrium dynamics, quantum resonances, spectral deformation.

\section{Introduction}

We consider an open quantum system $\mathrm{S}+\mathrm{R}$, where $\mathrm{S}$ is a "system of interest" and $\mathrm{R}$ is an "environment" or "reservoir". Typically, $\mathrm{S}$ is a system under examination in a laboratory, like an atom, a molecule or an aggregate of spins. It is not possible in reality to isolate any physical system entirely from its surroundings $R$, and only if we take these surroundings into account can we consider the total system as being closed and evolving according to a hamiltonian dynamics. The reservoir $\mathrm{R}$ is supposed to be a very large quantum system compared to S. An immediate question is how the reservoir influences the dynamics of the small system. Prime examples of effects $\mathrm{S}$ shows are thermalization and decoherence. The former means that, due to its interaction with $\mathrm{R}$, the system $\mathrm{S}$ is driven to the equilibrium state at the temperature of $\mathrm{R}$. The effect of decoherence is the subject of this contribution, and we will describe it in detail in the next section. 
Typically, we may assume that we know the (relatively simple) microscopic structure of $\mathrm{S}$, while our knowledge of $\mathrm{R}$ is limited to its macroscopic characterization. In other words, we shall assume the energy levels and corresponding states of $\mathrm{S}$ to be given to us, as well as the thermodynamic parameters of $\mathrm{R}$ (temperature, pressure, chemical potential...). We will always assume that the state space of $\mathrm{S}$ is finite-dimensional. Of course, in a true concrete analysis, we will also have to specify the reservoir $\mathrm{R}$ on a microscopic level. However, when studying the reduced dynamics of $\mathrm{S}$, only the macroscopic properties of $\mathrm{R}$ will be left in the description. The details of the environment do not play any role. In order to avoid introducing the microscopic structure of the environment, often an effective dynamics of the small system $\mathrm{S}$ is introduced - however, any trustworthy effective dynamics has to be derived from a full microscopic model, to which certain reduction and/or approximation schemes are applied (e.g. Born- or Born-Markov approximations). Our approach is to start off with a fully microscopic model of $\mathrm{S}+\mathrm{R}$, to eliminate

the degrees of freedom of $\mathrm{R}$ and to analyze the remaining reduced description of S. In this process, we do not employ any approximation, however, our results are perturbative in the strength of the coupling between $\mathrm{S}$ and $\mathrm{R}$.

We will take reservoirs to be spatially infinitely extended quantum systems. This is not merely a mathematical convenience, but rather a physical necessity that is linked to the very phenomena we want to describe. Indeed, if we try to keep the reservoir very large but finite, then irreversible physical processes will not take place. One can understand this easily heuristically, since for finite systems, hamiltonians have pure point spectrum, and so the dynamics will not drive the system to a final state. On the other hand, we may want to consider reservoirs which are just large, but maybe not infinitely extended, say an oven in a laboratory. The temporal behaviour of such systems is approximated by that of systems with infinitely extended reservoirs on time-scales which are large, but not too large, see e.g. [5].

We focus in this paper on the phenomenon of decoherence. A definition of decoherence is the vanishing of off-diagonal matrix elements of the reduced density matrix of S. A state given by a diagonal density matrix is characterized by classical probabilities, in the sense that averages of observables are obtained by weighing averages in specific states with given probabilities. The quantum nature is contained in the off-diagonal reduced density matrix elements, which are responsible for interference effects typical for quantum mechanics [15]. In this sense, a decohering system undergoes a transition from quantum to classical behaviour.

\section{Description of decoherence}

The pure states of $\mathrm{S}+\mathrm{R}$ are described by normalized vectors $\psi$ in the Hilbert space $\mathfrak{H}=\mathfrak{H}_{\mathrm{S}} \otimes \mathfrak{H}_{\mathrm{R}}$. An observable $A$ is a (self-adjoint) operator on $\mathfrak{H}$, its expectation value in the state $\psi$ is $\langle A\rangle=\langle\psi, A \psi\rangle$. The dynamics is determined by the 
hamiltonian (energy operator)

$$
H=H_{\mathrm{S}}+H_{\mathrm{R}}+\lambda v,
$$

where $H_{\mathrm{S}}$ and $H_{\mathrm{R}}$ are the hamiltonians of $\mathrm{S}$ and $\mathrm{R}, \lambda \in \mathbb{R}$ is a coupling constant and where $v$ represents the interaction between $\mathrm{S}$ and $\mathrm{R}$. The dynamical equation is the Schrödinger equation,

$$
\mathrm{i} \hbar \partial_{t} \psi=H \psi \text {. }
$$

We will set for convenience $\hbar=1$, so that the state vector evolves as $\psi_{t}=\mathrm{e}^{-\mathrm{i} t H} \psi_{0}$. Not all states can be represented by a single vector $\psi$. Mixed states are determined by density matrices $\rho$ on $\mathfrak{H}$. These are non-negative (self-adjoint) trace-class operators which are normalized as $\operatorname{Tr} \rho=1$. The average of an observable $A$ in the mixed state $\rho$ is given by $\langle A\rangle=\operatorname{Tr}(\rho A)$. To any density matrix $\rho$, we can associate normalized vectors $\psi_{n}$ and probabilities $p_{n}, n=1,2, \ldots$, s.t.

$$
\rho=\sum_{n=1}^{\infty} p_{n}\left|\psi_{n}\right\rangle\left\langle\psi_{n}\right|,
$$

where $\left|\psi_{n}\right\rangle\left\langle\psi_{n}\right|$ is the rank-one orthonormal projection onto $\mathbb{C} \psi_{n}$ (spectral decomposition of $\rho$ ). Since the evolution of $\psi_{n}$ is given by $\mathrm{e}^{-\mathrm{i} t H} \psi_{n}$, it follows from (2.1) that the density matrix $\rho$ evolves according to $\rho_{t}=e^{-\mathrm{i} t H} \rho_{0} \mathrm{e}^{\mathrm{i} t H}$.

Consider the system $\mathrm{S}$ to be finite-dimensional, $\mathfrak{H}_{\mathrm{S}}=\mathbb{C}^{N}$. An example of a mixed state of $\mathrm{S}$ is its equilibrium state at temperature $T=1 / \beta$, given by the density matrix $\rho_{\mathrm{S}, \beta} \propto \mathrm{e}^{-\beta H_{\mathrm{S}}}$. It is readily seen that one cannot find any vector $\psi \in \mathbb{C}^{N}$ representing this state, i.e., having the property that $\langle\psi, A \psi\rangle$ equals $\operatorname{Tr}\left(\rho_{\mathrm{S}, \beta} A\right)$ for all $A \in B\left(\mathfrak{H}_{\mathrm{S}}\right)$. However, by enlarging the Hilbert space, such a vector can be found: view $\sqrt{\rho_{\mathrm{S}, \beta}}$ as an element of the Hilbert space of HilbertSchmidt operators on $\mathfrak{H}_{\mathrm{S}}$ (this space is naturally isomorphic to $\mathfrak{H}_{\mathrm{S}} \otimes \mathfrak{H}_{\mathrm{S}}$ ). Then clearly $\langle A\rangle=\operatorname{Tr}\left(\rho_{\mathrm{S}, \beta} A\right)=\left\langle\sqrt{\rho_{\mathrm{S}, \beta}}, A \sqrt{\rho_{\mathrm{S}, \beta}}\right\rangle_{\mathrm{HS}}$, where $\langle\kappa, \sigma\rangle_{\mathrm{HS}}=\operatorname{Tr}\left(\kappa^{*} \sigma\right)$ is the inner product of Hilbert-Schmidt operators. ${ }^{1}$

As mentioned in the introduction, the system $\mathrm{R}$ is infinitely extended in space. Consequently, even if it has a finite energy density, the total energy $H_{\mathrm{R}}$ is not well defined (is infinite). In fact, it is not even clear which Hilbert space can describe states of the infinitely extended system $R$. One constructs the system $R$ via the thermodynamic limit. First, one takes a state $\rho_{\mathrm{R}, \Lambda}$ of the reservoir constrained to a box $\Lambda \subset \mathbb{R}^{3}$, with fixed thermodynamic properties (such as temperature, density etc.). For each finite $\Lambda$, one knows the Hilbert space and the state. (For instance, a quantum gas in a box $\Lambda$ is described by the Hilbert space $\oplus_{n \geq 0} L^{2}\left(\Lambda^{n}, \mathrm{~d}^{3 n} x\right)$ (Fock space), and since $\Lambda$ is finite, the energy operator has discrete spectrum, so the Gibbs-state density matrix is well defined.) Then the size of the box is made larger and larger, $\Lambda \uparrow \mathbb{R}^{3}$. This defines averages of (localized) observables $A$ in the infinitely extended state, $E(A)=\lim _{\Lambda \uparrow \mathbb{R}^{3}} \operatorname{Tr}\left(\rho_{\mathrm{R}, \Lambda} A\right)$. One can now try to find a Hilbert space $\mathfrak{H}_{\mathrm{R}}$ and a normalized vector $\psi_{\mathrm{R}} \in \mathfrak{H}_{\mathrm{R}}$ such that $E(A)=\left\langle\psi_{\mathrm{R}}, A \psi_{\mathrm{R}}\right\rangle$.

\footnotetext{
${ }^{1}$ This is a manifestation of a general fact: a state over a $C^{*}$-algebra can be represented by a vector state in a Hilbert space. This is the so-called Gelfand-Naimark-Segal representation [3].
} 
This is a difficult task in general, but explicit expressions for Hilbert spaces and vectors have been found in the important cases of infinitely extended ideal quantum gases in thermal equilibrium. ${ }^{2}$ We understand that this construction has been carried out, and that the state of $\mathrm{R}$ is represented on the Hilbert space $\mathfrak{H}_{\mathrm{R}}$ by a vector (or a density matrix). We give a more detailed explanation of this procedure at the end of this section. For now we carry on with a more qualitative discussion.

Given the density matrix of the total system, $\rho_{t}$, how can we extract the dynamics of S? Define the reduced density matrix of S by

$$
\bar{\rho}_{t}:=\operatorname{Tr}_{\mathrm{R}}\left(\rho_{t}\right),
$$

where the trace is taken over $\mathfrak{H}_{\mathrm{R}}$ only (partial trace). This is a density matrix on $\mathfrak{H}_{\mathrm{S}}$, and it satisfies

$$
\operatorname{Tr}_{\mathrm{S}}\left(\bar{\rho}_{t} A_{\mathrm{S}}\right)=\operatorname{Tr}_{\mathrm{S}+\mathrm{R}}\left(\rho_{t}\left(A_{\mathrm{S}} \otimes \mathbb{1}_{\mathrm{R}}\right)\right)
$$

for all obvservables $A_{\mathrm{S}} \in B\left(\mathfrak{H}_{\mathrm{S}}\right)$. The reduced density matrix contains all information to describe the evolution of expectation values of observables of $\mathrm{S}$ alone. The degrees of freedom of $\mathrm{R}$ and the effects of the interaction between $\mathrm{S}$ and $\mathrm{R}$ are encoded in $\bar{\rho}_{t}$, which acts on the Hilbert space of the system $\mathrm{S}$ only.

Let $\left\{\varphi_{j}\right\}_{j=1}^{N}$ be a fixed basis of $\mathfrak{H}_{\mathrm{S}}$ and denote the matrix elements of $\bar{\rho}_{t}$ as $\left[\bar{\rho}_{t}\right]_{m, n}:=\left\langle\varphi_{m}, \bar{\rho}_{t} \varphi_{n}\right\rangle$. A definition of decoherence is the vanishing of off-diagonal reduced density matrix elements in the limit of large times,

$$
\lim _{t \rightarrow \infty}\left[\bar{\rho}_{t}\right]_{m, n}=0, \quad \forall m \neq n .
$$

This is a basis dependent notion of disappearance of correlations,

$$
\bar{\rho}_{t}=\sum_{m, n} c_{m, n}(t)\left|\varphi_{m}\right\rangle\left\langle\varphi_{n}\left|\longrightarrow \sum_{m} p_{m}(t)\right| \varphi_{m}\right\rangle\left\langle\varphi_{m}\right|,
$$

as $t \rightarrow \infty$. Most often, the basis considered is the energy basis, consisting of eigenvectors of $H_{\mathrm{S}}$. A mixture of states $\varphi_{j}$ of the form $\sum_{m, n} c_{m, n}\left|\varphi_{m}\right\rangle\left\langle\varphi_{n}\right|$ is called an incoherent mixture if all "off-diagonals" vanish, $c_{m, n}=0$ for $m \neq n$. Else it is called a coherent mixture of the $\varphi_{j}$. The process (2.3) is thus a transition of a coherent to an incoherent mixture. Hence the name decoherence.

\subsection{An explicitly solvable model of decoherence}

Consider S to be an $N$-level system, coupled to a reservoir R of thermal bosons at temperature $T=1 / \beta$ through an energy-conserving interaction (see [14] for the qubit case, $N=2$, and [11] for general $N$ ).

The Hilbert space and hamiltonian of $\mathrm{S}$ are given by $\mathfrak{H}_{\mathrm{S}}=\mathbb{C}^{N}$ and $H_{\mathrm{S}}=$ $\operatorname{diag}\left(E_{1}, \ldots, E_{N}\right)$, respectively, and the interaction operator is $v=G \otimes \varphi(g)$, where $G=\operatorname{diag}\left(\gamma_{1}, \ldots, \gamma_{N}\right)$. Here,

$$
\varphi(g)=\frac{1}{\sqrt{2}}\left[a^{*}(g)+a(g)\right]
$$

\footnotetext{
${ }^{2}$ For bosons, this is known as the Araki-Woods construction, for fermions it is the Araki-Wyss construction, $[1,2,13]$.
} 
where the $a^{\#}(g)$ are the usual bosonic creation and annihilation operators, smeared out with a form factor $g \in L^{2}\left(\mathbb{R}^{3}, \mathrm{~d}^{3} k\right)$ (momentum space representation): $a(g)=$ $\int_{\mathbb{R}^{3}} \overline{g(k)} a(k) \mathrm{d}^{3} k, a^{*}(g)=\int_{\mathbb{R}^{3}} g(k) a^{*}(k) \mathrm{d}^{3} k,\left[a(k), a^{*}(l)\right]=\delta(k-l)$.

Since $\left[H_{\mathrm{S}}, H\right]=\left[H_{\mathrm{S}}, H_{\mathrm{S}}+H_{\mathrm{R}}+v\right]=0$ the energy of the small system is conserved. This model is exactly solvable. The solution is given by

$$
\left[\bar{\rho}_{t}\right]_{m, n}=\left[\bar{\rho}_{0}\right]_{m, n} \mathrm{e}^{-\mathrm{i} t\left(E_{m}-E_{n}\right)+\mathrm{i} \alpha_{m, n}(t)},
$$

where

$$
\begin{aligned}
\alpha_{m, n}(t) & =\left(\gamma_{m}^{2}-\gamma_{n}^{2}\right) S(t)+\mathrm{i}\left(\gamma_{m}-\gamma_{n}\right)^{2} \Gamma(t) \\
\Gamma(t) & =\int_{\mathbb{R}^{3}}|g(k)|^{2} \operatorname{coth}(\beta|k| / 2) \frac{\sin ^{2}(|k| t / 2)}{|k|^{2}} \mathrm{~d}^{3} k \\
S(t) & =\frac{1}{2} \int_{\mathbb{R}^{3}}|g(k)|^{2} \frac{|k| t-\sin (|k| t)}{|k|^{2}} \mathrm{~d}^{3} k .
\end{aligned}
$$

The parameter $\beta$ in the above expression for $\Gamma(t)$ is the inverse temperature of the reservoir. We immediately see that

1. The populations are constant, $\left[\bar{\rho}_{t}\right]_{m, m}=\left[\bar{\rho}_{0}\right]_{m, m}$ for all $m$ and all $t$.

2. If $\gamma_{m}=\gamma_{n}$ for some $m \neq n$, then the corresponding off-diagonal matrix element does not decay (decoherence-free subspaces).

3. Full decoherence (2.2) occurs if and only if $\Gamma(t) \rightarrow \infty$ as $t \rightarrow \infty$. Whether this happens or not depends on the infrared behaviour ( $s m a l l k$ ) of the form factor, as well as on the space dimension. Let the infrared behaviour be characterized by $g(k) \sim|k|^{p}$ as $|k| \sim 0$. We obtain in three space-dimensions

$$
\begin{aligned}
& \lim _{t \rightarrow \infty} \frac{\alpha_{m, n}(t)}{t}= \\
& \frac{1}{2}\left(\gamma_{m}^{2}-\gamma_{n}^{2}\right)\left\langle g,|k|^{-1} g\right\rangle+\mathrm{i}\left(\gamma_{m}-\gamma_{n}\right)^{2} \begin{cases}0 & \text { if } p>0 \\
\text { const. } & \text { if } p=-1 / 2 \\
+\infty & \text { if } p<-1 / 2\end{cases}
\end{aligned}
$$

For $p=-1 / 2$ the off-diagonal matrix elements decay exponentially quickly, $\left|\left[\bar{\rho}_{t}\right]_{m, n}\right| \sim \mathrm{e}^{- \text {const. } t\left(\gamma_{m}-\gamma_{n}\right)^{2}}$ and for $p<-1 / 2$ the decay is quicker. For $p>0$ the function $h(k):=|g(k)|^{2} \operatorname{coth}(\beta|k| / 2)|k|^{-2}$ is integrable on $\mathbb{R}^{3}$. We write $\sin ^{2}(|k| t / 2)=\frac{1}{2}(1-\cos (|k| t))$ and obtain from $(2.7)$

$$
\Gamma(t)=\frac{1}{2} \int_{\mathbb{R}^{3}} \frac{|g(k)|^{2}}{|k|^{2}} \operatorname{coth}(\beta|k| / 2) \mathrm{d}^{3} k-\operatorname{Re}(\widehat{F}(t)),
$$

where $\widehat{F}$ is the Fourier transform of the function

$$
F(r)=\frac{1}{4} \operatorname{coth}(\beta|r| / 2) \int_{S^{2}}|g(|r|, \sigma)|^{2} \mathrm{~d} \sigma,
$$


defined for and integrable on $r \in \mathbb{R}$ ( $\mathrm{d} \sigma$ is the uniform measure on the sphere $\left.S^{2}\right)$. From the Riemann-Lebesgue lemma we know that $\lim _{t \rightarrow \infty} \widehat{F}(t)=0$, so

$$
\lim _{t \rightarrow \infty} \Gamma(t)=\frac{1}{2} \int_{\mathbb{R}^{3}} \frac{|g(k)|^{2}}{|k|^{2}} \operatorname{coth}(\beta|k| / 2) \mathrm{d}^{3} k \neq 0, \quad \text { for } p>0 .
$$

That is, for $p>0$ we do not have full decoherence.

This is a non-demolition model $\left(H_{\mathrm{S}}\right.$ conserved), in which processes of absorption and emission of quanta of the reservoir by the system $\mathrm{S}$ are suppressed. To enable such processes one needs interactions $v$ which do not commute with $H_{\mathrm{S}}$. In the latter case, one typically expects that thermalization takes place. The phenomenon of thermalization can be described as follows.

Let $\rho(\beta, \lambda)$ be the equilibrium state of the total system at temperature $T=1 / \beta$ (where $\lambda$ is the coupling constant measuring the strength of interaction between $\mathrm{S}$ and $\mathrm{R}$ ) and let $\rho_{t=0}$ be any initial density matrix (on $\mathfrak{H}$ ). Thermalization means that

$$
\operatorname{Tr}_{\mathrm{S}+\mathrm{R}}\left(\rho_{t} A\right) \longrightarrow \operatorname{Tr}_{\mathrm{S}+\mathrm{R}}(\rho(\beta, \lambda) A), \text { as } t \rightarrow \infty,
$$

where $A$ is any observable of the total system $\mathrm{S}+\mathrm{R}$. The convergence (2.9) implies that

$$
\bar{\rho}_{t} \longrightarrow \bar{\rho}_{\infty}(\beta, \lambda):=\operatorname{Tr}_{\mathrm{R}}(\bar{\rho}(\beta, \lambda)),
$$

as $t \rightarrow \infty$. An expansion of $\bar{\rho}_{\infty}(\beta, \lambda)$ in the coupling constant $\lambda$ gives

$$
\bar{\rho}_{\infty}(\beta, \lambda)=\bar{\rho}_{\infty}(\beta, 0)+O(\lambda),
$$

where $\bar{\rho}_{\infty}(\beta, 0)$ is the Gibbs state of the system S. The Gibbs state (density matrix) is diagonal in the energy basis (diagonalizing $H_{\mathrm{S}}$ ), however, the correction term $O(\lambda)$ is not, in general (see e.g. [11] for explicit calculations for the qubit). This shows the following effect.

Even if $\mathrm{S}$ is initially in an incoherent superposition of energy eigenstates it will acquire some "residual coherence" of order $O(\lambda)$ in the process of thermalization. This leads us to defining decoherence in thermalizing systems as being the decay of off-diagonals of $\bar{\rho}_{t}$ to their (non-zero) limit values, i.e., to the corresponding off-diagonals of $\bar{\rho}_{\infty}(\beta, \lambda)$.

In examining the vast literature on this topic (some references are $[7,14,15$, 16]) we have only encountered either models with energy-conserving interactions (which are explicitly solvable), or models with markovian approximations with uncontrolled errors (master equations, Lindblad dynamics). The goal of our work is to describe decoherence for systems which may also exhibit thermalization, in a rigorous fashion (controlled perturbation expansion). 


\subsection{Description of the infinitely extended reservoir $R$}

Before taking the thermodynamic limit, as outlined above, the reservoir confined to a box $\Lambda$ is described by the bosonic Fock space

$$
\mathfrak{H}_{\mathrm{R}, \Lambda}=\bigoplus_{n \geq 0} L_{\mathrm{sym}}^{2}\left(\Lambda^{n}, \mathrm{~d}^{3 n} x\right)
$$

where the subindex "sym" means that we take symmetric square-integrable functions only (indistinguishable Bose particles). The hamiltonian is that of noninteracting particles, given by $H_{\mathrm{R}, \Lambda}=\oplus_{n \geq 0} H_{\mathrm{R}, \Lambda}^{(n)}$, with $H_{\mathrm{R}, \Lambda}^{(n)}=\sum_{j=1}^{n} \sqrt{-\partial_{x_{j}}^{2}}$ (with periodic boundary conditions). The density matrix $\rho_{\mathrm{R}, \beta, \Lambda}=Z_{\mathrm{R}, \beta, \Lambda}^{-1} \mathrm{e}^{-\beta H_{\mathrm{R}, \Lambda}}$ is a well defined trace-class operator on the space (2.10), and the normalization factor $Z_{\mathrm{R}, \beta, \Lambda}$ is chosen so that $\operatorname{Tr}\left(\rho_{\mathrm{R}, \beta, \Lambda}\right)=1$. One calculates $[1,13] E_{\beta}\left(a^{\#}(f)\right):=$ $\lim _{\Lambda \uparrow \mathbb{R}^{3}} \operatorname{Tr}\left(\rho_{\mathrm{R}, \beta, \Lambda} a^{\#}(f)\right)=0$, where $a^{\#}$ stands for either $a$ or $a^{*}$, and

$$
E_{\beta}\left(a^{*}(f) a(g)\right):=\lim _{\Lambda \uparrow \mathbb{R}^{3}} \operatorname{Tr}\left(\rho_{\mathrm{R}, \beta, \Lambda} a^{*}(f) a(g)\right)=\left\langle g, \frac{1}{\mathrm{e}^{\beta|k|}-1} f\right\rangle,
$$

where the square-integrable $f, g$ are represented in Fourier transform in the inner product on the right hand side. ${ }^{3}$ All products of creation and annihilation operators can be calculated using the Wick theorem [3], so (2.11) (plus the vanishing of averages of $a(f)$ and $\left.a^{*}(f)\right)$ determines the infinitely extended thermal state $E_{\beta}$ of $\mathrm{R}$ completely. We consider here only reservoir equilibrium states below critical density, i.e., in absence of Bose-Einstein condensate.

The Araki-Woods Hilbert space representation is given by

$$
\mathfrak{H}_{\mathrm{R}}=\mathcal{F} \otimes \mathcal{F}
$$

where $\mathcal{F}=\oplus_{n \geq 0} L_{\text {sym }}^{2}\left(\mathbb{R}^{3 n}, \mathrm{~d}^{3 n} x\right)$,

$$
\psi_{\mathrm{R}}=\Omega \otimes \Omega,
$$

the product of the Fock vacua in $\mathcal{F}$, and

$$
a_{\beta}^{*}(g)=a^{*}\left(\frac{e^{\beta|k|}}{\mathrm{e}^{\beta|k|}-1} g\right) \otimes \mathbb{1}+\mathbb{1} \otimes a\left(\frac{1}{\mathrm{e}^{\beta|k|}-1} \bar{g}\right),
$$

where $\bar{g}$ is the complex conjugate of $g$, and where the $a^{\#}$ are the ordinary Fock creation and annihilation operators on $\mathcal{F}$. We also set $a_{\beta}(g):=\left[a_{\beta}^{*}(g)\right]^{*}$. It is easy to check that

$$
E_{\beta}\left(a^{*}(f) a(g)\right)=\left\langle\psi_{\mathrm{R}}, a_{\beta}^{*}(f) a_{\beta}(g) \psi_{\mathrm{R}}\right\rangle .
$$

This last equation shows us that we have successfully represented the thermal state of the infinitely extended $\mathrm{R}$ as a vector state on a concrete Hilbert space.

\footnotetext{
${ }^{3}$ Of course, one has to restrict this to functions for which the r.h.s. is well defined.
} 


\section{Dynamical resonance theory: Results}

Let $\mathrm{S}$ be an $N$-level system, $\mathfrak{H}_{\mathrm{S}}=\mathbb{C}^{N}$, with energies $\left\{E_{j}\right\}_{j=1}^{N}$, and let $\mathrm{R}$ be the free massless Bose field, spatially infinitely extended in $\mathbb{R}^{3}$ in equilibrium at temperature $T=1 / \beta$, as described at the end of Section 2 .

The interaction operator is obtained by taking the thermodynamic limit of $\lambda v_{\Lambda}=\lambda G \otimes \varphi\left(g_{\Lambda}\right)$. Here, $G=G^{*} \in B\left(\mathfrak{H}_{\mathrm{S}}\right)$ is a self-adjoint $N \times N$ matrix and $\varphi\left(g_{\Lambda}\right)$ is the smoothed-out field operator (2.4) acting on $\mathfrak{H}_{\mathrm{R}, \Lambda},(2.10)$, and $g_{\Lambda}(x)=\chi_{\Lambda}(x) g(x)$ is the function $g$, cut off by being set equal to zero outside $\Lambda$. It is customary to abbreviate this description by simply writing

$$
v=\lambda G \otimes \varphi(g),
$$

and the thermodynamic limit is understood to be taken automatically.

We denote the average of observables $A \in B\left(\mathfrak{H}_{\mathrm{S}}\right)$ at time $t$ by

$$
\langle A\rangle_{t}:=\operatorname{Tr}_{\mathrm{S}}\left(\bar{\rho}_{t} A\right),
$$

and the ergodic average is denoted by

$$
\left\langle\langle A\rangle_{\infty}:=\lim _{T \rightarrow \infty} \frac{1}{T} \int_{0}^{T}\langle A\rangle_{t} \mathrm{~d} t .\right.
$$

Our approach is based on a dynamical resonance theory, where resonances are treated in a setting of spectral deformation (see Section 4). This leads to the following regularity requirement which we assume to be fulfilled throughout this paper.

(A) The function

$$
g_{\beta}(u, \sigma):=\sqrt{\frac{u}{1-\mathrm{e}^{-\beta u}}}|u|^{1 / 2} \begin{cases}g(u, \sigma) & \text { if } u \geq 0 \\ \mathrm{e}^{\mathrm{i} \phi} \bar{g}(-u, \sigma) & \text { if } u<0\end{cases}
$$

is such that $\vartheta \mapsto g_{\beta}(u+\vartheta, \sigma)$ has an analytic continuation, as a map $\mathbb{C} \rightarrow$ $L^{2}\left(\mathbb{R} \times S^{2}, \mathrm{~d} u \times \mathrm{d} \sigma\right)$, into $\{|\vartheta|<\tau\}$, for some $\tau>0$. Here, $\phi$ is an arbitrary fixed phase. (See [6] for the usefulness and physical interpretation of this phase.)

Examples of admissible $g$ are $g(k)=g_{1}(\sigma)|k|^{p} \mathrm{e}^{-|k|^{2}}$, where $p=-1 / 2+n, n=$ $0,1,2, \ldots$, and $g_{1}(\sigma)=\mathrm{e}^{\mathrm{i} \phi} \bar{g}_{1}(\sigma)$. They include the physically most important cases, see also [14]. We point out that it is possible to weaken condition (A) considerably, at the expense of a mathematically more involved treatment, as mentioned in $[11,12]$. The following result is the main result of [11]. We give an outline of the proof in Section 4.

Theorem 3.1 (Evolution of observables [11]). There is a $\lambda_{0}>0$ s.t. the following statements hold for $|\lambda|<\lambda_{0}, t \geq 0$, and $A \in B\left(\mathfrak{H}_{\mathrm{S}}\right)$.

1. $\langle\langle A\rangle\rangle_{\infty}$ exists.

2. We have

$$
\langle A\rangle_{t}-\langle\langle A\rangle\rangle_{\infty}=\sum_{\varepsilon \neq 0} \mathrm{e}^{\mathrm{i} t \varepsilon} R_{\varepsilon}(A)+O\left(\lambda^{2} \mathrm{e}^{-\tau t}\right),
$$


where the $\varepsilon$ are "resonance energies", $0 \leq \operatorname{Im} \varepsilon<\tau / 2$, and $R_{\varepsilon}(A)$ are linear functionals of $A$ which depend on the initial state $\rho_{t=0}$.

3. Let $e$ be an eigenvalue of the operator $H_{\mathrm{S}} \otimes \mathbb{1}_{\mathrm{S}}-\mathbb{1}_{\mathrm{S}} \otimes H_{\mathrm{S}}$ (acting on $\mathfrak{H}_{\mathrm{S}} \otimes \mathfrak{H}_{\mathrm{S}}$ ). For $\lambda=0$ each $\varepsilon$ coincides with one of the $e$ and we have the following expansion for small $\lambda$

$$
\varepsilon \equiv \varepsilon_{e}^{(s)}=e-\lambda^{2} \delta_{e}^{(s)}+O\left(\lambda^{4}\right) .
$$

The $\delta_{e}^{(s)}$ satisfy $\operatorname{Im} \delta_{e}^{(s)} \leq 0$. They are eigenvalues of so-called level shift operators $\Lambda_{e}$, and $s=1, \ldots, \nu(s) \leq \operatorname{mult}(e)$ labels the eigenvalue splitting. Furthermore, we have

$$
R_{\varepsilon}(A)=\sum_{(m, n) \in I_{e}} \kappa_{m, n} A_{m, n}+O\left(\lambda^{2}\right),
$$

with $I_{e}=\left\{(m, n) \mid E_{m}-E_{n}=e\right\}$, and where $A_{m, n}$ is the (m,n)-matrix element of $A$ and the numbers $\kappa_{m, n}$ depend on the initial state.

Discussion. Relation (3.3) gives a detailed picture of the dynamics of averages of observables. The resonance energies $\varepsilon$ and the functionals $R_{\varepsilon}$ can be calculated for concrete models, to arbitrary precision (in the sense of rigorous perturbation theory in $\lambda$ ). See Section 3.1 for explicit expressions for the qubit. In the absence of interaction $(\lambda=0)$ we have $\varepsilon=e \in \mathbb{R}$. Depending on the interaction each resonance energy $\varepsilon$ may migrate into the upper complex plane, or it may stay on the real axis, as $\lambda \neq 0$. The averages $\langle A\rangle_{t}$ approach their ergodic means $\left\langle\langle A\rangle_{\infty}\right.$ if and only if $\operatorname{Im} \varepsilon>0$ for all $\varepsilon \neq 0$. In this case the convergence takes place on the time scale $[\operatorname{Im} \varepsilon]^{-1}$. Otherwise $\langle A\rangle_{t}$ oscillates. A sufficient condition for decay is that $\operatorname{Im} \delta_{e}^{(s)}<0$ (and $\lambda$ small).

There are two kinds of processes which drive the decay: energy-exchange processes and energy preserving ones. The former are induced by interactions enabling processes of absorption and emission of field quanta with energies corresponding to the Bohr frequencies of S (this is the "Fermi Golden Rule Condition"). Energy preserving interactions suppress such processes, allowing only for a phase change of the system during the evolution ("phase damping").

Even if the initial density matrix, $\rho_{t=0}$, is a product of the system and reservoir density matrices, the density matrix $\rho_{t}$ at any subsequent moment of time $t>0$ is not of product form. The evolution creates entanglement between the system and reservoir. Our technique does not require $\rho_{t=0}$ to be a product state [11].

Our next goal is to use Theorem 3.1 to describe in detail the decay of reduced density matrix elements. According to Theorem 3.1 the dynamics is governed by the resonance energies $\varepsilon_{e}^{(s)}$ whose lowest order contributions $\delta_{e}^{(s)}$ are eigenvalues of level shift operators $\Lambda_{e}$. In what follows we assume that all eigenvalues $\delta_{e}^{(s)}$ are simple. We denote the corresponding eigenvector by $\eta_{e}^{(s)}$, and the eigenvector associated to the adjoint operator $\Lambda_{e}^{*}$ with eigenvalue $\overline{\delta_{e}^{(s)}}$ is denoted by $\widetilde{\eta}_{e}^{(s)}$. They 
are normalized as $\left\langle\eta_{e}^{(s)}, \widetilde{\eta}_{e}^{(s)}\right\rangle=1$. The assumption of simplicity of the spectrum of $\Lambda_{e}$ is not necessary at all for our method, it is simply made to make the exposition somewhat simpler. Let $\left\{\varphi_{n}\right\}$ be an orthornomal basis of $\mathbb{C}^{N}$ diagonalizing the Hamiltonian of S, $H_{\mathrm{S}} \varphi_{n}=E_{n} \varphi_{n}$. The matrix element $\left[\bar{\rho}_{t}\right]_{m, n}$ is obtained by choosing the observable $A=\left|\varphi_{n}\right\rangle\left\langle\varphi_{m}\right|$ in (3.2). We denote the difference of two eigenvalues of $H_{\mathrm{S}}$ by $E_{m, n}=E_{m}-E_{n}$. A closer analysis of the functionals $R_{\varepsilon}$ yields the following result, the proof of which we give in Section 5.

Theorem 3.2 (Dominant dynamics). There is a constant $\lambda_{1}$ s.t. if $0<|\lambda|<\lambda_{1}$, then for all $m, n$ and all $t \geq 0$

$$
\begin{aligned}
& {\left[\bar{\rho}_{t}\right]_{m, n}-\left\langle\left\langle\mid \varphi_{n}\right\rangle\left\langle\varphi_{m} \mid\right\rangle\right\rangle_{\infty}=} \\
& \sum_{\left\{s: \varepsilon_{E_{n, m}}^{(s)} \neq 0\right\}} \mathrm{e}^{\mathrm{i} \varepsilon_{E_{n, m}}^{(s)}} \sum_{\left\{k, l: E_{l, k}=E_{n, m}\right\}} \sigma_{m, n ; k, l}^{(s)}\left[\bar{\rho}_{0}\right]_{k, l}+O\left(\lambda^{2} \mathrm{e}^{-t \gamma_{m, n}}\right),
\end{aligned}
$$

where $\gamma_{m, n}=\min \left\{\operatorname{Im} \varepsilon_{e}^{(s)}: \varepsilon_{e}^{(s)} \neq 0\right.$ and $\left.e \neq E_{n, m}\right\}$. The mixing constants $\sigma_{m, n ; k, l}^{(s)}$ are given by

$$
\sigma_{m, n ; k, l}^{(s)}=\left\langle\widetilde{\eta}_{E_{n, m}}^{(s)}, \varphi_{n} \otimes \varphi_{m}\right\rangle\left\langle\varphi_{l} \otimes \varphi_{k}, \eta_{E_{n, m}}^{(s)}\right\rangle
$$

$\eta_{E_{n, m}}^{(s)}$ and $\widetilde{\eta}_{E_{n, m}}^{(s)}$ being the resonance eigenvectors introduced above.

Discussion. The group of matrix elements $\left[\bar{\rho}_{t}\right]_{m, n}$ associated to the same energy difference $e=E_{n}-E_{m}$ evolve in a coupled way, while groups belonging to different $e$ evolve indpendently, in the regime of Theorem 3.2. It is clear that the eigenvalue $e=0$ is always degenerate $\left(\varphi_{k} \otimes \varphi_{k}\right.$ is always an associated eigenvector, for all $k)$. One easily sees that if $e=E_{n}-E_{m}$ is simple then $\sigma_{m, n ; k, l}^{(s)}$ vanishes unless $(k, l)=(m, n)$, in which case $\sigma_{m, n ; k, l}^{(s)}=1$ equals one (this follows simply from the fact that $\eta_{e}$ and $\widetilde{\eta}_{e}$ belong to the spectral subspace associated to $e$ ). The main term of the r.h.s. of (3.5) is then simply $\mathrm{e}^{\mathrm{i} t \varepsilon_{E_{n, m}}}$, so

$$
\left[\bar{\rho}_{t}\right]_{m, n}-\left\langle\left\langle\mid \varphi_{n}\right\rangle\left\langle\varphi_{m} \mid\right\rangle\right\rangle_{\infty}=\mathrm{e}^{\mathrm{i} t \varepsilon_{E_{n, m}, m}}\left[\bar{\rho}_{0}\right]_{m, n}+O\left(\lambda^{2} \mathrm{e}^{-t \gamma_{m, n}}\right) .
$$

The usefulness of Theorem 3.2 is that it relates $\left[\bar{\rho}_{t}\right]_{m, n}$ to the initial conditions $\left[\bar{\rho}_{0}\right]_{k, l}$. We can understand how to arrive at Theorem 3.2 from Theorem 3.1 in the following way. The expansion (3.4) implies that the main term of $R_{\varepsilon}\left(\left|\varphi_{n}\right\rangle\left\langle\varphi_{m}\right|\right)$ is non-vanishing only if $\varepsilon$ bifurcates out of $e=E_{n}-E_{m}$. This means that all contributions to the sum in (3.3) with $\varepsilon$ not bifurcating out of $E_{n}-E_{m}$ are of order $\lambda^{2}$, and decaying according to $\mathrm{e}^{\mathrm{i} t \varepsilon}$. These terms, plus the $O\left(\lambda^{2} \mathrm{e}^{-\tau t}\right)$ term in (3.3), constitute the remainder term in (3.5).

The constants $\gamma_{m, n}$ are typically of order $\lambda^{2}$ (they may be of higher order if the so-called Fermi Golden Rule condition for efficient coupling is not satisfied [9]). Expansion (3.5) is thus useful in the regime

$$
\lambda^{2} \mathrm{e}^{-t \lambda^{2} \min \left\{\operatorname{Im} \delta_{e}^{(s)}: e \neq E_{n, m}\right\}} \ll e^{-t \lambda^{2} \max \left\{\operatorname{Im} \delta_{E_{n, m}}^{(s)}: s=1, \ldots, \nu\left(E_{n, m}\right)\right\}} .
$$


In other words, given any finite maximal time of interest $t_{\max }$, there is a $\lambda_{1}$ s.t. if $0<|\lambda|<\lambda_{1}$, expansion (3.5) is valid, and the remainder term is negligeable for all $0 \leq t \leq t_{\max }$. The expansion (3.5) thus isolates the dominant dynamics.

\subsection{Application: thermalization versus decoherence time for a qubit}

A qubit, or spin $1 / 2$, is described by the Hilbert-space of pure states $\mathbb{C}^{2}$. The hamiltonian is $H_{\mathrm{S}}=\operatorname{diag}\left(E_{1}, E_{2}\right)$ (in the canonical basis of $\left.\mathbb{C}^{2}\right)$. We set $\Delta=$ $E_{2}-E_{1}>0$. The coupling operator is given by the self-adjoint operator

$$
v=\left[\begin{array}{ll}
a & c \\
\bar{c} & b
\end{array}\right] \otimes \varphi(g),
$$

where $\varphi(g)$ is given in (2.4). The operator $H_{\mathrm{S}} \otimes \mathbb{1}-\mathbb{1} \otimes H_{\mathrm{S}}$ has four eigenvalues, $e \in\{-\Delta, 0,0, \Delta\}$. One calculates the resonance energies associated to these $e$ to be (see also Theorem 3.1 and the next subsection)

$$
\begin{aligned}
\varepsilon_{0}^{(1)}(\lambda) & =0 \\
\varepsilon_{0}^{(2)}(\lambda) & =\mathrm{i} \lambda^{2}|c|^{2} \xi(\Delta)+O\left(\lambda^{4}\right) \\
\varepsilon_{\Delta}(\lambda) & =\Delta+\lambda^{2} R+\frac{\mathrm{i}}{2} \lambda^{2}\left[|c|^{2} \xi(\Delta)+(b-a)^{2} \xi(0)\right]+O\left(\lambda^{4}\right) \\
\varepsilon_{-\Delta}(\lambda) & =-\overline{\varepsilon_{\Delta}(\lambda)}
\end{aligned}
$$

where we have set

$$
\xi(\eta):=\pi \int_{\mathbb{R}^{3}} \operatorname{coth}\left(\frac{\beta|k|}{2}\right)|g(k)|^{2} \delta(\eta-|k|) \mathrm{d}^{3} k
$$

and (P.V. denoting the principal value)

$$
R=\frac{b^{2}-a^{2}}{2}\left\langle g,|k|^{-1} g\right\rangle+\frac{|c|^{2}}{2} \mathrm{P} . \mathrm{V} \cdot \int_{\mathbb{R} \times S^{2}} u^{2} \operatorname{coth}\left(\frac{\beta|k|}{2}\right) \frac{|g(|u|, \sigma)|^{2}}{u-\Delta} \mathrm{d} u \mathrm{~d} \sigma .
$$

The corresponding resonance eigenvectors (defined before Theorem 3.2) are as follows, where $\left\{\varphi_{1}, \varphi_{2}\right\}$ is the canonical orthonormal basis of $\mathbb{C}^{2}$, and where $\varphi_{i, j}=$ $\varphi_{i} \otimes \varphi_{j}:$

$$
\begin{gathered}
\eta_{0}^{(1)}=\varphi_{1,1}+\varphi_{2,2}, \quad \widetilde{\eta}_{0}^{(1)}=\frac{1}{1+\mathrm{e}^{-\beta \Delta}}\left[\varphi_{1,1}+\mathrm{e}^{-\beta \Delta} \varphi_{2,2}\right], \\
\eta_{0}^{(2)}=\varphi_{1,1}-\mathrm{e}^{\beta \Delta} \varphi_{2,2}, \quad \widetilde{\eta}_{0}^{(2)}=\frac{1}{1+\mathrm{e}^{\beta \Delta}}\left[\varphi_{1,1}-\varphi_{2,2}\right],
\end{gathered}
$$


and $\eta_{\Delta}=\widetilde{\eta}_{\Delta}=\varphi_{2,1}, \eta_{-\Delta}=\widetilde{\eta}_{\Delta}=\varphi_{1,2}$. Note that $\eta_{0}^{(1)}$ is just the (not normalized) trace state on S. The mixing constants $\sigma_{m, n ; k, l}^{(s)}$ (see Theorem 3.2) are thus

$$
\begin{aligned}
\sigma_{1,2 ; 1,2}^{(1)}=\sigma_{2,1 ; 2,1}^{(1)} & =1 \\
\sigma_{1,1 ; 1,1}^{(2)} & =\left\langle\eta_{0}^{(2)}, \varphi_{1,1}\right\rangle\left\langle\varphi_{1,1}, \eta_{0}^{(2)}\right\rangle=\frac{1}{1+\mathrm{e}^{\beta \Delta}}, \\
\sigma_{1,1 ; 2,2}^{(2)}=\sigma_{2,2 ; 1,1}^{(2)} & =\left\langle\eta_{0}^{(2)}, \varphi_{1,1}\right\rangle\left\langle\varphi_{2,2}, \eta_{0}^{(2)}\right\rangle=\frac{-1}{1+\mathrm{e}^{-\beta \Delta}}, \\
\sigma_{2,2 ; 2,2}^{(2)} & =\left\langle\eta_{0}^{(2)}, \varphi_{2,2}\right\rangle\left\langle\varphi_{2,2}, \eta_{0}^{(2)}\right\rangle=\frac{1}{1+\mathrm{e}^{-\beta \Delta}} .
\end{aligned}
$$

We shall assume that the Fermi Golden Rule is satisfied: $\xi(\Delta) \neq 0$. Then zero is a simple resonance eigenvalue, $\varepsilon_{0}^{(1)}=0$, and consequently, for $e=0$ the term $s=1$ is not present in the sum (3.5). Theorem 3.1 thus gives the following dominant dynamics:

$$
\begin{aligned}
& {\left[\bar{\rho}_{t}\right]_{1,1}-\left\langle\left\langle\mid \varphi_{1}\right\rangle\left\langle\varphi_{1} \mid\right\rangle\right\rangle_{\infty} \sim \mathrm{e}^{\mathrm{i} t \varepsilon_{0}^{(2)}(\lambda)}\left\{\frac{\left[\bar{\rho}_{0}\right]_{1,1}}{1+\mathrm{e}^{\beta \Delta}}-\frac{\left[\bar{\rho}_{0}\right]_{2,2}}{1+\mathrm{e}^{-\beta \Delta}}\right\},} \\
& {\left[\bar{\rho}_{t}\right]_{1,2}-\left\langle\left\langle\mid \varphi_{2}\right\rangle\left\langle\varphi_{1} \mid\right\rangle\right\rangle_{\infty} \sim \mathrm{e}^{\mathrm{i} t \varepsilon_{\Delta}(\lambda)}\left[\bar{\rho}_{0}\right]_{1,2} .}
\end{aligned}
$$

The dynamics for $\left[\bar{\rho}_{t}\right]_{2,2}$ and $\left[\bar{\rho}_{t}\right]_{2,1}$ are easily obtained also directly from Theorem 3.1 , or by using that $\left[\bar{\rho}_{t}\right]_{1,1}+\left[\bar{\rho}_{t}\right]_{2,2}=1\left(\right.$ since $\left.\operatorname{Tr} \bar{\rho}_{t}=1\right)$ and the fact that $\bar{\rho}_{t}$ is selfadjoint. We point out that since the system $\mathrm{S}+\mathrm{R}$ approaches its (joint) equilibrium as $t \rightarrow \infty$, we have $\left\langle\left\langle\mid \varphi_{1}\right\rangle\left\langle\varphi_{1} \mid\right\rangle\right\rangle_{\infty}=\frac{\mathrm{e}^{\beta \Delta}}{1+\mathrm{e}^{\beta \Delta}}+O\left(\lambda^{2}\right)$ and $\left\langle\left\langle\mid \varphi_{2}\right\rangle\left\langle\varphi_{1} \mid\right\rangle\right\rangle_{\infty}=O\left(\lambda^{2}\right)$ (Gibbs distribution). This law can also be recovered by setting $t=0$ in (3.7), (3.8) and using that $\left[\bar{\rho}_{0}\right]_{2,2}=1-\left[\bar{\rho}_{0}\right]_{1,1}$.

The thermalization time (decay of diagonals) is $\tau_{\text {th }}:=\left[\operatorname{Im} \varepsilon_{0}^{(2)}(\lambda)\right]^{-1}$, and the decoherence time (decay of off-diagonals) is $\tau_{\mathrm{dec}}:=\left[\operatorname{Im} \varepsilon_{\Delta}(\lambda)\right]^{-1}$. Their ratio is

$$
\frac{\tau_{\mathrm{th}}}{\tau_{\mathrm{dec}}}=\frac{1}{2}\left[1+\frac{(b-a)^{2}}{|c|^{2}} \frac{\xi(0)}{\xi(\Delta)}\right]+O\left(\lambda^{2}\right)
$$

Note that we have $\xi(0)>0$ for infra-red behaviour $g(k) \sim|k|^{-1 / 2}$ as $|k| \sim 0$ and $\xi(0)=0$ for more regular infra-red behaviour. Moreover, $\xi(0) \sim T$ and $\xi(\Delta) \sim$ const. $>0$, as the temperature $T \sim 0$.

Spin-Boson model. The Hamiltonian of $\mathrm{S}$ is given by $[4,8]$

$$
H_{\mathrm{S}}=-\frac{1}{2} \hbar \Delta_{0} \sigma_{x}+\frac{1}{2} \epsilon \sigma_{z}
$$

where the $\sigma$ are Pauli matrices, $\Delta_{0}$ is the bare tunneling matrix element, and $\epsilon$ is the bias. The coupling operator is

$$
v=\sigma_{z} \otimes \varphi(g) .
$$


This determines the matrix elements $a, b, c$ in the general formulation, and we obtain

$$
\frac{(b-a)^{2}}{|c|^{2}}=16 \frac{\epsilon^{2}}{\hbar^{2} \Delta_{0}^{2}} .
$$

This shows for instance that the thermalization time will become smaller relative to the decoherence time if the bias $\epsilon$ is decreased, or if the tunneling parameter $\Delta_{0}$ is increased.

Explicit form of the level shift operators. For the sake of completeness, we include the explicit form of the level shift operators $\Lambda_{e}, e=0, \pm \Delta$. By definition,

$$
\Lambda_{e}=P_{e} I \bar{P}_{e}\left(\bar{L}_{0}-e+\mathrm{i} 0\right)^{-1} \bar{P}_{e} I P_{e},
$$

where $P_{e}$ is the spectral projection onto the eigenspace of $H_{\mathrm{S}} \otimes 1-11 \otimes H_{\mathrm{S}}$ associated to the eigenvalue $e, \bar{P}_{e}=1-P_{e}, \bar{L}_{0}$ is the operator $L_{0}$ restricted to $\operatorname{Ran} \bar{P}_{e}$, and where $I$ is the interaction operator, see [11] and Section 4. The explicit form of $\Lambda_{e}$ has been calculated in [11] for a general $N$-level system coupled to the thermal bose environment (Proposition 5.1 of [11]). ${ }^{4}$ The explicit form of $\Lambda_{0}$, expressed in the basis $\left\{\varphi_{1} \otimes \varphi_{1}, \varphi_{2} \otimes \varphi_{2}\right\}$ of $\operatorname{Ran} P_{0}$ is

$$
\Lambda_{0}=\frac{\mathrm{i}}{2} \frac{|c|^{2} \xi(\Delta)}{\mathrm{e}^{\beta \Delta}-1}\left[\begin{array}{cc}
1 & -1 \\
-e^{\beta \Delta} & \mathrm{e}^{\beta \Delta}
\end{array}\right]
$$

The dimension of $\operatorname{Ran} P_{ \pm \Delta}$ is one, so $\Lambda_{ \pm \Delta}$ reduces simply to a number,

$$
\Lambda_{ \pm \Delta}=R \pm \frac{\mathrm{i}}{2}\left[|c|^{2} \xi(\Delta)+(b-a)^{2} \xi(0)\right],
$$

where $R$ is given in (3.6). Knowing the explicit form of the level shift operators, the expansions of the resonance energies and resonance eigenvectors are now easy to obtain.

\section{Outline of resonance approach}

Consider any observable $A \in B\left(\mathfrak{H}_{\mathrm{S}}\right)$. We have

$$
\begin{aligned}
\langle A\rangle_{t} & =\operatorname{Tr}_{\mathrm{S}}\left[\bar{\rho}_{t} A\right] \\
& =\operatorname{Tr}_{\mathrm{S}+\mathrm{R}}\left[\rho_{t} A \otimes \mathbb{1}_{\mathrm{R}}\right] \\
& =\left\langle\psi_{0}, \mathrm{e}^{\mathrm{i} t L_{\lambda}}\left[A \otimes \mathbb{1}_{\mathrm{S}} \otimes \mathbb{1}_{\mathrm{R}}\right] \mathrm{e}^{-\mathrm{i} t L_{\lambda}} \psi_{0}\right\rangle .
\end{aligned}
$$

In the last step, we pass to the representation Hilbert space of the system (the GNS Hilbert space), where the initial density matrix is represented by the vector $\psi_{0}$ (in particular, the Hilbert space of the small system becomes $\mathfrak{H}_{\mathrm{S}} \otimes \mathfrak{H}_{\mathrm{S}}$ ), see also after equation (2.1) and Section 2.2. For this outline we take the initial state to be one represented by the product vector $\psi_{0}=\psi_{\mathrm{S}, \infty} \otimes \psi_{\mathrm{R}}$, where $\psi_{\mathrm{S}, \infty}$ is the trace

\footnotetext{
${ }^{4}$ In the present work, we take the generator of dynamics to be the Liouville operator associated to the reference state $\psi_{0}=\psi_{\mathrm{S}, \infty} \otimes \psi_{\mathrm{R}}$, see Section 4. In [11] the Liouville operator is taken with respect to the reference vector $\psi_{\mathrm{S}, \beta} \otimes \psi_{\mathrm{R}}$. Those two choices are related by a simple transformation, and all physical results are independent of the particular choice of reference state.
} 
state of $\mathrm{S},\left\langle\psi_{\mathrm{S}, \infty},\left(A_{\mathrm{S}} \otimes \mathbb{1}_{\mathrm{S}}\right) \psi_{\mathrm{S}, \infty}\right\rangle=\frac{1}{N} \operatorname{Tr}\left(A_{\mathrm{S}}\right)$, and where $\psi_{\mathrm{R}}$ is the equilibrium state of $\mathrm{R}$ at a fixed inverse temperature $0<\beta<\infty$, (2.13). (This form of the initial state is is not necessary for our method to work, see [11].) The dynamics is implemented by the group of automorphisms $\mathrm{e}^{\mathrm{i} t L_{\lambda}} \cdot \mathrm{e}^{-\mathrm{i} t L_{\lambda}}$. The self-adjoint generator $L_{\lambda}$ is called the Liouville operator. It is of the form $L_{\lambda}=L_{0}+\lambda W$, where $L_{0}=L_{\mathrm{S}}+L_{\mathrm{R}}$ represents the uncoupled Liouville operator, and $\lambda W$ is the interaction (represented in the GNS Hilbert space).

We borrow a trick from the analysis of open systems far from equilibrium: there is a (non-self-adjoint) generator $K_{\lambda}$ s.t.

$$
\begin{aligned}
\mathrm{e}^{\mathrm{i} t L_{\lambda}} A \mathrm{e}^{-\mathrm{i} t L_{\lambda}} & =\mathrm{e}^{\mathrm{i} t K_{\lambda}} A \mathrm{e}^{-\mathrm{i} t K_{\lambda}} \text { for all observables } A, t \geq 0 \text {, and } \\
K_{\lambda} \psi_{0} & =0 .
\end{aligned}
$$

There is a standard way of constructing $K_{\lambda}$ given $L_{\lambda}$ and the reference vector $\psi_{0} . K_{\lambda}$ is of the form $K_{\lambda}=L_{0}+\lambda I$, where the interaction term undergoes a certain modification ( $W \rightarrow I$ ), c.f. [11]. As a consequence, formally, we may replace the propagators in (4.1) by those involving $K$. The resulting propagator which is directly applied to $\psi_{0}$ will then just disappear due to the invariance of $\psi_{0}$. One can carry out this procedure in a rigorous manner, obtaining the following resolvent representation [11]

$$
\langle A\rangle_{t}=-\frac{1}{2 \pi \mathrm{i}} \int_{\mathbb{R}-\mathrm{i}}\left\langle\psi_{0},\left(K_{\lambda}(\omega)-z\right)^{-1}\left[A \otimes \mathbb{1}_{\mathrm{S}} \otimes \mathbb{1}_{\mathrm{R}}\right] \psi_{0}\right\rangle \mathrm{e}^{\mathrm{i} t z} \mathrm{~d} z,
$$

where $K_{\lambda}(\omega)=L_{0}(\omega)+\lambda I(\omega), I$ is representing the interaction, and $\omega \mapsto K_{\lambda}(\omega)$ is a spectral deformation (translation) of $K_{\lambda}$. The latter is constructed as follows. There is a deformation transformation $U(\omega)=\mathrm{e}^{-\mathrm{i} \omega D}$, where $D$ is the (explicit) self-adjoint generator of translations $[11,10]$ transforming the operator $K_{\lambda}$ as

$$
K_{\lambda}(\omega)=U(\omega) K_{\lambda} U(\omega)^{-1}=L_{0}+\omega N+\lambda I(\omega) .
$$

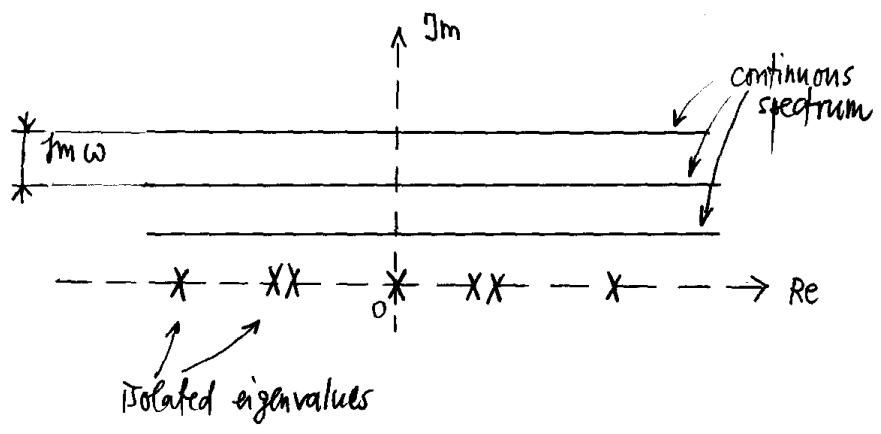

Figure 1. Spectrum of $K_{0}(\omega)$ 
Here, $N=N_{1} \otimes \mathbb{1}+\mathbb{1} \otimes N_{1}$ is the total number operator of $\mathfrak{H}_{\mathrm{R}},(2.12)$, and where $N_{1}$ is the usual number operator on $\mathcal{F}$. $N$ has spectrum $\mathbb{N} \cup\{0\}$, where 0 is a simple eigenvalue (vacuum eigenvector $\psi_{\mathrm{R}}$ ). For real values of $\omega, U(\omega)$ is a group of unitaries. The spectrum of $K_{\lambda}(\omega)$ depends on $\operatorname{Im} \omega$ and moves according to the value of $\operatorname{Im} \omega$, whence the name "spectral deformation". Even though $U(\omega)$ becomes unbounded for complex $\omega$, the r.h.s. of (4.3) is a well defined closed operator on a dense domain, analytic in $\omega$ at zero. Analyticity is used in the derivation of (4.2) and this is where the analyticity condition (A) before Theorem 3.1 comes into play. The operator $I(\omega)$ is infinitesimally small with respect to the number operator $N$. Hence we use perturbation theory in $\lambda$ to examine the spectrum of $K_{\lambda}(\omega)$.

The point of the spectral deformation is that the (important part of the) spectrum of $K_{\lambda}(\omega)$ is much easier to analyze than that of $K_{\lambda}$, because the deformation uncovers the resonances of $K_{\lambda}$. We have (see Figure 1)

$$
\operatorname{spec}\left(K_{0}(\omega)\right)=\left\{E_{i}-E_{j}\right\}_{i, j=1, \ldots, N} \bigcup_{n \geq 1}\{\omega n+\mathbb{R}\},
$$

because $K_{0}(\omega)=L_{0}+\omega N, L_{0}$ and $N$ commute, and the eigenvectors of $L_{0}=$ $L_{\mathrm{S}}+L_{\mathrm{R}}$ are $\varphi_{i} \otimes \varphi_{j} \otimes \psi_{\mathrm{R}}$. The continuous spectrum is bounded away from the isolated eigenvalues by a gap of size $\operatorname{Im} \omega$. For values of the coupling parameter $\lambda$ small compared to $\operatorname{Im} \omega$, we can follow the displacements of the eigenvalues by using analytic perturbation theory. (Note that for $\operatorname{Im} \omega=0$, the eigenvalues are imbedded into the continuous spectrum, and analytic perturbation theory is not valid!

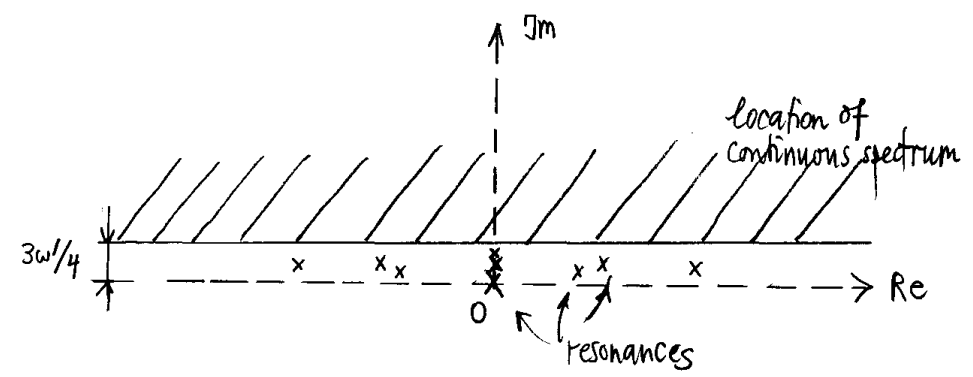

Figure 2. Spectrum of $K_{\lambda}(\omega)$. Resonances $\varepsilon_{e}^{(s)}$ are uncovered.

Theorem 4.1. (See Fig. 2.) Fix $\operatorname{Im} \omega$ s.t. $0<\operatorname{Im} \omega<\tau$ (where $\tau$ is as in Condition (A) given after (3.2)). There is a constant $c_{0}>0$ s.t. if $|\lambda| \leq c_{0} / \beta$ then, for all $\omega$ with $\operatorname{Im} \omega>\omega^{\prime}$, the spectrum of $K_{\lambda}(\omega)$ in the complex half-plane $\left\{\operatorname{Im} z<\omega^{\prime} / 2\right\}$ is independent of $\omega$ and consists purely of the distinct eigenvalues

$$
\left\{\varepsilon_{e}^{(s)}: e \in \operatorname{spec}\left(L_{\mathrm{S}}\right), s=1, \ldots, \nu(e)\right\},
$$


where $1 \leq \nu(e) \leq \operatorname{mult}(e)$ counts the splitting of the eigenvalue $e$. Moreover, $\lim _{\lambda \rightarrow 0}\left|\varepsilon_{e}^{(s)}(\lambda)-e\right|=0$ for all $s$, and we have $\operatorname{Im} \varepsilon_{e}^{(s)} \geq 0$. Also, the continuous spectrum of $K_{\lambda}(\omega)$ lies in the region $\left\{\operatorname{Im} z \geq 3 \omega^{\prime} / 4\right\}$.

Next we separate the contributions to the path integral in (4.2) coming from the singularities at the resonance energies and from the continuous spectrum. We deform the path of integration $z=\mathbb{R}-\mathrm{i}$ into the line $z=\mathbb{R}+\mathrm{i} \omega^{\prime} / 2$, thereby picking up the residues of poles of the integrand at $\varepsilon_{e}^{(s)}$ (all $\left.e, s\right)$. Let $\mathcal{C}_{e}^{(s)}$ be a small circle around $\varepsilon_{e}^{(s)}$, not enclosing or touching any other spectrum of $K_{\lambda}(\omega)$. We introduce the generally non-orthogonal Riesz spectral projections

$$
Q_{e}^{(s)}=Q_{e}^{(s)}(\omega, \lambda)=-\frac{1}{2 \pi \mathrm{i}} \int_{\mathcal{C}_{e}^{(s)}}\left(K_{\lambda}(\omega)-z\right)^{-1} \mathrm{~d} z .
$$

It follows from (4.2) that

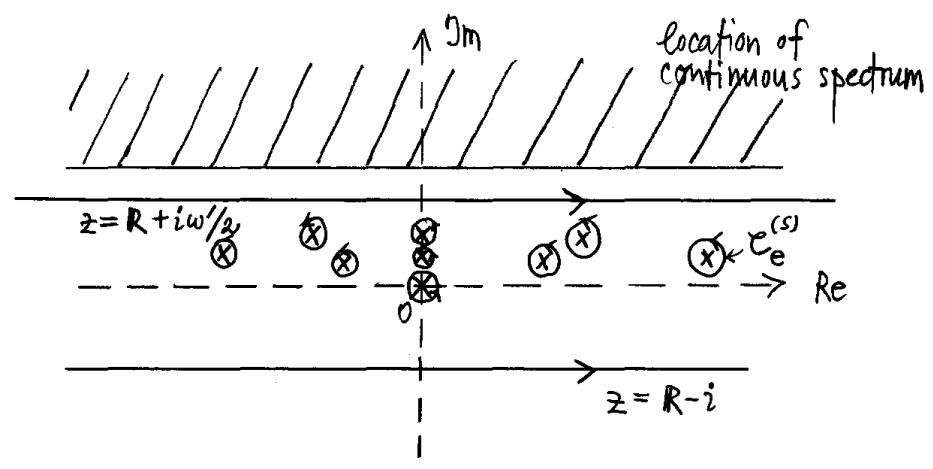

FIGURE 3. Contour deformation: $\int_{\mathbb{R}-\mathrm{i}} \mathrm{d} z=\sum_{e, s} \int_{\mathcal{C}_{e}^{(s)}} \mathrm{d} z+\int_{\mathbb{R}+\mathrm{i} \omega^{\prime} / 2} \mathrm{~d} z$

$$
\langle A\rangle_{t}=\sum_{e} \sum_{s=1}^{\nu(e)} \mathrm{e}^{\mathrm{i} t \varepsilon_{e}^{(s)}}\left\langle\psi_{0}, Q_{e}^{(s)}\left[A \otimes \mathbb{1}_{\mathrm{S}} \otimes \mathbb{1}_{\mathrm{R}}\right] \psi_{0}\right\rangle+O\left(\lambda^{2} \mathrm{e}^{-\omega^{\prime} t / 2}\right) .
$$

Note that the imaginary parts of all resonance energies $\varepsilon_{e}^{(s)}$ are smaller than $\omega^{\prime} / 2$, so that the remainder term in (4.5) is not only small in $\lambda$, but it also decays faster than all of the terms in the sum! (See also Figure 3.)

Finally, we notice that all terms in $(4.5)$ with $\varepsilon_{e}^{(s)} \neq 0$ will vanish in the ergodic mean limit, so

$$
\langle\langle A\rangle\rangle_{\infty}=\lim _{T \rightarrow \infty} \frac{1}{T} \int_{0}^{T}\langle A\rangle_{t} \mathrm{~d} t=\sum_{s: \varepsilon_{0}^{(s)}=0}\left\langle\psi_{0}, Q_{0}^{(s)}\left[A \otimes \mathbb{1}_{\mathrm{R}} \otimes \mathbb{1}_{\mathrm{R}}\right] \psi_{0}\right\rangle .
$$

The identification of the linear functionals

$$
R_{\varepsilon_{e}^{(s)}}(A)=\left\langle\psi_{0}, Q_{e}^{(s)}\left[A \otimes \mathbb{1}_{\mathrm{S}} \otimes \mathbb{1}_{\mathrm{R}}\right] \psi_{0}\right\rangle
$$


(c.f. (3.3)) is useful for concrete calculations, as well as in the proof of Theorem 3.2. This concludes the outline of the proof of Theorem 3.1.

\section{Proof of Theorem 3.2}

The proof is based on expansion (3.3) together with formula (4.6). We have $\left[\bar{\rho}_{t}\right]_{m, n}=\operatorname{Tr}\left(\bar{\rho}_{t}\left|\varphi_{n}\right\rangle\left\langle\varphi_{m}\right|\right)$, and it follows that

$$
\begin{aligned}
& {\left[\bar{\rho}_{t}\right]_{m, n}-\left\langle\left\langle\mid \varphi_{n}\right\rangle\left\langle\varphi_{m} \mid\right\rangle\right\rangle_{\infty}} \\
& \quad=\sum_{\left\{e, s: \varepsilon_{e}^{(s)} \neq 0\right\}} \mathrm{e}^{\mathrm{i} t \varepsilon_{e}^{(s)}}\left\langle\psi_{0}, Q_{e}^{(s)}\left[\left|\varphi_{n}\right\rangle\left\langle\varphi_{m}\right| \otimes \mathbb{1}_{\mathrm{S}}\right] \psi_{0}\right\rangle+O\left(\lambda^{2} \mathrm{e}^{-\omega^{\prime} t / 2}\right) .
\end{aligned}
$$

We leave out the trivial $\mathbb{1}_{\mathrm{R}}$. Remembering that $\psi_{0}=\psi_{\mathrm{S}, \infty} \otimes \psi_{\mathrm{R}}$, where $\psi_{\mathrm{S}, \infty}$ is the trace state of $\mathrm{S}$, represented by the vector $\frac{1}{\sqrt{N}} \sum_{j=1}^{N} \varphi_{j} \otimes \varphi_{j}$, we see that

$$
\left[\left|\varphi_{n}\right\rangle\left\langle\varphi_{m}\right| \otimes \mathbb{1}_{\mathrm{S}}\right] \psi_{0}=\frac{1}{\sqrt{N}} \varphi_{n} \otimes \varphi_{m} \otimes \psi_{\mathrm{R}}
$$

We shall treat in here the case where all resonance eigenvalues $\varepsilon_{e}^{(s)}$ are simple (the general case is dealt with in a similar fashion). Thus $Q_{e}^{(s)}=\left|\chi_{e}^{(s)}\right\rangle\left\langle\widetilde{\chi}_{e}^{(s)}\right|$ is a rankone projection, with $K_{\lambda}(\omega) \chi_{e}^{(s)}=\varepsilon_{e}^{(s)} \chi_{e}^{(s)}, K_{\lambda}(\omega)^{*} \widetilde{\chi}_{e}^{(s)}=\overline{\varepsilon_{e}^{(s)}} \widetilde{\chi}_{e}^{(s)}$ and with the normalization $\left\langle\chi_{e}^{(s)}, \tilde{\chi}_{e}^{(s)}\right\rangle=1$. We expand the resonance eigenvectors in powers of $\lambda$,

$$
\chi_{e}^{(s)}=\eta_{e}^{(s)} \otimes \psi_{\mathrm{R}}+O(\lambda), \quad \tilde{\chi}_{e}^{(s)}=\widetilde{\eta}_{e}^{(s)} \otimes \psi_{\mathrm{R}}+O(\lambda),
$$

where $\eta_{e}^{(s)}, \widetilde{\eta}_{e}^{(s)}$ are eigenvectors of the level shift operator $\Lambda_{e}$ associated to the eigenvalue $\delta_{e}^{(s)}$ and its complex conjugate, respectively (see also before Theorem 3.2 and [11]). $\Lambda_{e}$ acts on the eigenspace $P\left(L_{\mathrm{S}}=e\right)$, and $\eta_{e}^{(s)}, \widetilde{\eta}_{e}^{(s)} \in \operatorname{Ran} P\left(L_{\mathrm{S}}=e\right)$. We obtain

$$
Q_{e}^{(s)}=\left|\eta_{e}^{(s)}\right\rangle\left\langle\widetilde{\eta}_{e}^{(s)}|\otimes| \psi_{\mathrm{R}}\right\rangle\left\langle\psi_{\mathrm{R}}\right|+R_{1}(\lambda)
$$

where $R_{1}$ satisfies $\left\langle\psi_{\mathrm{R}}\left|R_{1}(\lambda)\right| \psi_{\mathrm{R}}\right\rangle=O\left(\lambda^{2}\right)$. (This term is of order $\lambda^{2}$ and not only $\lambda$ since the average of the interaction (3.1) vanishes in the vacuum state.) Combining (5.2) and (5.3) and setting $\varphi_{m, n}=\varphi_{m} \otimes \varphi_{n}$, we arrive at

$$
\begin{aligned}
& \left\langle\psi_{0}, Q_{e}^{(s)}\left[\left|\varphi_{n}\right\rangle\left\langle\varphi_{m}\right| \otimes \mathbb{1}_{\mathrm{S}}\right] \psi_{0}\right\rangle \\
& =\frac{\delta_{e=E_{n, m}}}{\sqrt{N}}\left\langle\psi_{0}, \eta_{e}^{(s)} \otimes \psi_{\mathrm{R}}\right\rangle\left\langle\widetilde{\eta}_{e}^{(s)}, \varphi_{n, m}\right\rangle+O\left(\lambda^{2}\right) \\
& =\frac{\delta_{e=E_{n, m}}}{\sqrt{N}} \sum_{\left\{l, k: E_{l, k}=e\right\}}\left\langle\psi_{0}, \varphi_{l, k} \otimes \psi_{\mathrm{R}}\right\rangle\left\langle\varphi_{l, k}, \eta_{e}^{(s)}\right\rangle\left\langle\widetilde{\eta}_{e}^{(s)}, \varphi_{n, m}\right\rangle+O\left(\lambda^{2}\right) .
\end{aligned}
$$

(The $\delta$ is the Kronecker delta here.) The initial values are recovered from the first scalar product on the r.h.s.,

$$
\frac{1}{\sqrt{N}}\left\langle\psi_{0}, \varphi_{l, k} \otimes \psi_{\mathrm{R}}\right\rangle=\left\langle\psi_{0},\left[\left|\varphi_{l}\right\rangle\left\langle\varphi_{k}\right| \otimes \mathbb{1}_{\mathrm{S}}\right] \psi_{0}\right\rangle=\left[\bar{\rho}_{0}\right]_{k, l} .
$$


This shows that

$$
\left\langle\psi_{0}, Q_{e}^{(s)}\left[\left|\varphi_{n}\right\rangle\left\langle\varphi_{m}\right| \otimes \mathbb{1}_{\mathrm{S}}\right] \psi_{0}\right\rangle=\delta_{e, E_{n, m}} \sum_{\left\{l, k: E_{l, k}=E_{n, m}\right\}} \sigma_{m, n ; k, l}\left[\bar{\rho}_{0}\right]_{k, l}+O\left(\lambda^{2}\right),
$$

where the "mixing coefficients" $\sigma_{m, n ; k, l}^{(s)}$ are defined in Theorem 3.2. We use expression (5.4) in (5.1),

$$
\begin{aligned}
& {\left[\bar{\rho}_{t}\right]_{m, n}-\left\langle\left\langle\mid \varphi_{n}\right\rangle\left\langle\varphi_{m} \mid\right\rangle\right\rangle_{\infty}} \\
& \quad=\sum_{\left\{e, s: \varepsilon_{e}^{(s)} \neq 0\right\}} \mathrm{e}^{\mathrm{i} t \varepsilon_{e}^{(s)}}\left[\delta_{e, E_{n, m}} \sum_{\left\{l, k: E_{l, k}=E_{n, m}\right\}} \sigma_{m, n ; k, l}^{(s)}\left[\bar{\rho}_{0}\right]_{k, l}+O\left(\lambda^{2}\right)\right]+O\left(\lambda^{2} \mathrm{e}^{-\omega^{\prime} t / 2}\right) .
\end{aligned}
$$

The main term in the sum selects $e=E_{n, m}$ and only the summation over $s$ : $\varepsilon_{E_{n, m}}^{(s)} \neq 0$ remains. This yields the dominant part in the r.h.s. of formula (3.5). The remainder is

$$
\sum_{\left\{e: e \neq E_{n, m}, s: \varepsilon_{e}^{(s)} \neq 0\right\}} \mathrm{e}^{\mathrm{i} t \varepsilon_{e}^{(s)}} O\left(\lambda^{2}\right)+O\left(\lambda^{2} \mathrm{e}^{-\omega^{\prime} t / 2}\right),
$$

which is $O\left(\lambda^{2} \mathrm{e}^{-t \gamma_{m, n}}\right)$, as indicated in Theorem 3.2. This concludes the proof of Theorem 3.2.

\section{References}

[1] Araki, H., Woods, E.J.: Representations of the canonical commutation relations describing a nonrelativistic infinite free Bose gas J. Math. Phys., 4 637-662 (1963)

[2] Araki, H., Wyss, W.: Representations for canonical anticommutation relations. Helv. Phys. Acta 37 136-159 (1964)

[3] Bratteli, O., Robinson, D.W., Operator Algebras and Quantum Statistical Mechanics I, II. Texts and Monographs in Physics, Springer-Verlag, 1987

[4] Caldeira, A.O., Leggett, A.J.: Quantum tunnelling in a dissipative system. Ann. Phys. 149, 374-456 (1983)

[5] Fröhlich, J., Merkli, M., Ueltschi, D.: Dissipative transport: thermal contacts and tunneling junctions. Ann. Henri Poincaré 4, no.5, 897-945 (2003)

[6] Fröhlich, J., Merkli, M.: Another return of "Return to Equilibrium". Commun. Math. Phys. 251, 235-262 (2004)

[7] Joos, E., Zeh, H.D., Kiefer, C., Giulini, D., Kupsch, J. Stamatescu, I.O.: Decoherence and the appearence of a classical world in quantum theory. Second edition. Springer Verlag, Berlin, 2003

[8] Leggett, A.J., Chakravarty, S., Dorsey, A.T., Fisher, M.P.A., Garg, A., Zwerger, W.: Dynamics of the dissipative two-state system. Rev. Mod. Phys. 59, no.1, 1-85 (1987)

[9] Merkli, M.: Level shift operators for open quantum systems. J. Math. Anal. Appl. 327 no. 1, 376-399 (2007)

[10] Merkli, M., Mück, M., Sigal, I.M.: Instability of equilibrium states for coupled heat reservoirs at different temperatures, J. Funct. Anal. 243, Issue 1, 310-344 (2006) 
[11] Merkli, M., Sigal, I.M., Berman, G.P.: Resonance theory of dechoherence and termalization, Ann. Phys. (2007), doi:10.1016/j.aop.2007.04013

[12] Merkli, M., Sigal, I.M., Berman, G.P.: Decoherence and thermalization, Phys. Rev. Lett. 98, no. 13, 130401, 4pp. (2007)

[13] Merkli, M.: The Ideal Quantum Gas. Open quantum systems. I, 183-233, Lecture Notes in Math., 1880, Springer, Berlin, 2006

[14] Palma, M.G., Suominen, K.-A., Ekert, A.: Quantum computers and dissipation, Proc. R. Soc. Lond. A 452, 567-584 (1996)

[15] Schlosshauer, M., Decoherence and the quantum-to-classical transition, The frontiers collection, Springer Verlag, 2007

[16] Zurek, W.H.: Decoherence, einselection, and the quantum origins of the classical. Rev. Mod. Phys. 75, 715 - 775 (2003)

\author{
Marco Merkli \\ Department of Mathematics and Statistics \\ Memorial University of Newfoundland \\ St. John's, NL \\ Canada, A1C 5S7 \\ e-mail: merkli@math.mun.ca
}

